\title{
Local treatment of rhabdomyosarcoma of the female genital tract: Expert consensus from the Children's Oncology Group (COG), European Soft Tissue Sarcoma Group (EpSSG), and the Cooperative Weichteilsarkom Studiengruppe (CWS)
}

Timothy Lautz ${ }^{1}$, Helene Martelli ${ }^{2}$, Joerg Fuchs ${ }^{3}$, Cyrus Chargari ${ }^{4}$, Naima Smeulders ${ }^{5}$, Candace Granberg ${ }^{6}$, Suzanne Wolden ${ }^{7}$, Monika Sparber-Sauer ${ }^{8}$, Douglas Hawkins ${ }^{9}$, Gianni Bisogno $^{10}$, Ewa Koscielniak ${ }^{8}$, David A. Rodeberg ${ }^{11}$, and Guido Seitz ${ }^{12}$

${ }^{1}$ Ann and Robert H Lurie Children's Hospital of Chicago

${ }^{2}$ Hopital Bicetre

${ }^{3}$ University of Tuebingen

${ }^{4}$ Institut de Cancerologie Gustave Roussy

${ }^{5}$ Great Ormond Street Hospital for Children

${ }^{6}$ Mayo Clinic

${ }^{7}$ Memorial Sloan Kettering Cancer Center

${ }^{8}$ Klinikum Stuttgart Olgahospital Frauenklinik

${ }^{9}$ Seattle Children's Hospital

${ }^{10}$ Division of Hematology/Oncology

${ }^{11}$ East Carolina University

${ }^{12}$ University Children's Hospital Marburg

July 1, 2020

\begin{abstract}
Background The International Soft Tissue Sarcoma Consortium (INSTRuCT) was founded as an international collaboration between different pediatric soft tissue sarcoma cooperative groups (COG, EpSSG, CWS). Besides other tasks, a major goal of the INSTRuCT is to develop consensus expert opinions for best clinical treatment. This consensus paper for patients with rhabdomyosarcoma of the female genital tract (FGU-RMS) provides treatment recommendations for local treatment, long term follow up and fertility preservation. Methods Review of the current literature was combined with recommendations of the treatment protocols of the appropriate clinical trials. Additionally, opinions of international FGU-RMS experts were incorporated into recommendations. Results The prognosis of FGU-RMS is favorable with an excellent response to chemotherapy. Initial complete surgical resection is not indicated, but diagnosis should be established properly. In patients with tumors localized at the vagina or cervix demonstrating incomplete response after induction chemotherapy, local radiotherapy (brachytherapy) should be carried out. In patients with persistent tumors at the corpus uteri, hysterectomy should be performed. Fertility preservation should be considered in all patients. Conclusion For the first time, an international consensus for the treatment of FGU-RMS patients could be achieved, which will help to harmonize the treatment in different study groups.
\end{abstract}

\section{Abbreviations}




\begin{tabular}{ll}
\hline BPRMS & Bladder-prostate rhabdomyosarcoma \\
\hline BT & Brachytherapy \\
CED & Cyclophosphamide equivalent dose \\
COG & Children's Oncology Group \\
CR & Complete response \\
CWS & Cooperative Weichteilsarkom Studiengruppe \\
EBRT & External beam radiotherapy \\
EpSSG & European pediatric Soft Tissue Sarcoma Study Group \\
ES & Event free survival \\
FGU & Female genito-urinary \\
HDR & High dose rate \\
INSTRuCT & International Soft Tissue Sarcoma Consortium \\
IRS & Intergroup Rhabdomyosarcoma Study Group \\
LDR & Low dose rate \\
MMT & Malignant Mesenchymal Tumor Group \\
OS & Overall survival \\
OTC & Ovarian tissue cryopreservation \\
PDR & Pulse-dose-rate \\
PR & Partial response \\
PRE & Primary re-excision \\
RMS & Rhabdomyosarcoma \\
RT & Radiotherapy \\
SIOP & International Society of Pediatric Oncology \\
STSC & Italian Soft Tissue Sarcoma Committee \\
\hline
\end{tabular}

Abstract

\section{Background}

The International Soft Tissue Sarcoma Consortium (INSTRuCT) was founded as an international collaboration between different pediatric soft tissue sarcoma cooperative groups (COG, EpSSG, CWS). Besides other tasks, a major goal of the INSTRuCT is to develop consensus expert opinions for best clinical treatment. This consensus paper for patients with rhabdomyosarcoma of the female genital tract (FGU-RMS) provides treatment recommendations for local treatment, long term follow up and fertility preservation.

\section{Methods}

Review of the current literature was combined with recommendations of the treatment protocols of the appropriate clinical trials. Additionally, opinions of international FGU-RMS experts were incorporated into recommendations.

\section{Results}

The prognosis of FGU-RMS is favorable with an excellent response to chemotherapy. Initial complete surgical resection is not indicated, but diagnosis should be established properly. In patients with tumors localized at the vagina or cervix demonstrating incomplete response after induction chemotherapy, local radiotherapy (brachytherapy) should be carried out. In patients with persistent tumors at the corpus uteri, hysterectomy should be performed. Fertility preservation should be considered in all patients.

\section{Conclusion}

For the first time, an international consensus for the treatment of FGU-RMS patients could be achieved, which will help to harmonize the treatment of these patients in different study groups.

\section{Introduction}


Rhabdomyosarcoma (RMS) is the most common pediatric soft tissue sarcoma accounting for $2 / 3$ of all sarcomas and approximately $7-8 \%$ of all solid malignant tumors in children. ${ }^{1}$ The genitourinary tract is the second most common primary tumor location. ${ }^{2}$ Tumors at this site are conventionally classified as bladderprostate RMS (BPRMS) and genitourinary non-bladder prostate RMS. Although perineal and perianal rhabdomyosarcoma have a close anatomical relationship in the lower pelvis, they are considered separately. Therefore, genitourinary non-bladder prostate RMS include tumors located at the paratesticular site or in the female genital tract (vulva, vagina and / or uterus, (FGU-RMS) which is the focus of this consensus statement).

Tumors of the female genital tract account for $4 \%$ of all RMS cases and $10 \%$ of all cases in females. ${ }^{3,4}$ Histology of most tumors is FOXO1 fusion negative embryonal RMS. ${ }^{5}$ The prognosis for FGU-RMS is favorable, with 5-year overall survival rates of more than $90 \% .^{4,6-8}$ In addition, tumors located at this site seem to respond extremely well to chemotherapy. ${ }^{7}$ There has been a paradigm shift in treatment from aggressive local control, with radical surgery and external beam radiotherapy (EBRT), towards more conservative approaches with chemotherapy alone in patients with complete response or organ-sparing surgery in combination with intracavitary brachytherapy (BT) or EBRT. This strategy has been introduced by the Malignant Mesenchymal Tumor Group (MMT) trials of the International Society of Pediatric Oncology (SIOP) ${ }^{4,7}$ and is currently used as the standard approach for patients treated within the European pediatric Soft Tissue Sarcoma Group (EpSSG). ${ }^{4}$ The Children's Oncology Group (COG) previously investigated an even more conservative strategy, employing lower doses of cyclophosphamide and omitting RT in complete responders. Due to increased rates of local recurrence on D9602 and ARST0331 for vaginal primary tumors, the COG now recommends both treatment with more intensive chemotherapy and the use of RT or brachytherapy for incompletely resected RMS of the vagina. ${ }^{8}$

Analysis of pooled data from four cooperative groups, including SIOP MMT, EpSSG, COG, and the Italian Soft Tissue Sarcoma Committee (STSC) examined the optimal treatment for patients with FGU-RMS. ${ }^{4}$ Two-hundred and thirty-seven patients were included in the analysis, with a 10-year event free survival rate (ESF) of $74 \%$ and a 10-year overall survival rate of $92 \%$. Patients in whom RT was initially omitted had inferior EFS but no difference in OS. The local control rate with BT is excellent. ${ }^{7}$ The pooled analysis concluded that half of the patients could be cured without external beam RT or radical surgery. ${ }^{4}$

A collaboration of the American and European pediatric oncology cooperative groups (COG, EpSSG and CWS) was initiated recently. The resulting International Soft Tissue Sarcoma Consortium (INSTRuCT) has several goals, one of which is to develop consensus positions regarding best practice treatment that would be broadly applicable. This consensus for patients with FGU-RMS provides treatment recommendations for the local treatment as well as long term follow up of these tumors.

\section{Rhabdomyosarcoma of the vagina}

RMS of the vagina are often fusion-negative, botryoid histology, which grow into the vaginal cavity, protruding outside the vulva and often with multifocal implantations. Fusion-positive alveolar histology and tumor invasion of adjacent structures are uncommon. ${ }^{4}$ These tumors demonstrate a good response to chemotherapy.

\section{Primary surgery}

\section{Expert consensus}

Initial investigations include MRI imaging of the pelvis, cystoscopy and vaginoscopy. These examinations should be carried out under general anesthesia and include a bimanual recto-vaginal examination. During these procedures, biopsies should be taken, which can be achieved by polypectomy or incisional biopsy without resection of the vaginal wall. Care should be taken to collect an adequate amount of tissue during the procedure in order to establish the diagnosis and for further molecular or genetic analysis. Complete surgical resection of vaginal tumors prior to chemotherapy is often impossible and unnecessary. The good response to chemotherapy precludes the need for aggressive upfront surgical resection. Therefore, primary tumor resection should be avoided with the exception of very small, localized and well circumscribed tumors 
that can be grossly excised with minimal compromise to normal local structures.

Spread to regional lymph nodes (inguinal lymph nodes) is extremely uncommon. Therefore, routine surgical lymph node evaluation is not recommended. Suspicious lymph nodes at initial imaging should be evaluated surgically during primary surgery/biopsy.

\section{Evidence}

The surgical approach for patients with FGU-RMS changed dramatically from the North American IRS-I trial, in which the primary surgery was commonly pelvic exenteration, hysterectomy or vaginectomy, towards preservation of these organs in IRS-III \& -IV. With these less radical procedures the excellent survival rates of IRS-I were preserved with much lower morbidity. ${ }^{6}$ Arndt et al. further outlined the evolution of treatment from IRS I-IV to determine the optimal management strategy for FGU-RMS, demonstrating 5-year failurefree and overall survival of $72 \%$ and $87 \%$, respectively, in those with nonmetastatic tumors. ${ }^{9}$ Importantly, aggressive initial surgical resection was performed in IRS-I but was discouraged by IRS-III and -IV to decrease morbidity. Rate of organ removal (vagina +/- uterus +/- bladder) decreased from $66 \%$ in IRS-I/II to $41 \%$ in IRS-III/IV, yet cure rates were preserved at $82 \%$ and $84 \%$, respectively. Of 27 patients with recurrence, $23(85 \%)$ were local/regional, and most did not receive any or had inadequate RT. Eighteen $(67 \%)$ were salvaged with additional multimodal treatments. As such, conservative initial surgery followed by primary chemotherapy approach with selective RT was recommended to decrease morbidity and late effects. This treatment paradigm was utilized in the subsequent series of COG trials D9602 and ARST0331. The majority of patients therefore had unresected disease at enrollment in both of these trials, however there was a significantly increased local recurrence rate between D9602 $(26 \%)$ study and the subsequent ARST0331 (43\%) likely due to decreasing the cumulative dose or eliminating cyclophosphamide and delaying or eliminating RT. ${ }^{8}$ Although high salvage rates allowed 3-year OS to remain excellent (95\%) on ARST0331, this was at the expense of intensified second-line therapy for patients who developed recurrence, with inherent long-term complications.

Further support for a conservative primary surgery paradigm came from a pooled, multinational analysis including data from 237 patients treated within trials of COG, SIOP MMT, STSC, and EpSSG in which approximately $50 \%$ of the patients achieved CR with chemotherapy alone and some were cured without systematic RT or radical surgery. ${ }^{4}$ In this large patient cohort only $31 \%(72 / 231)$ underwent upfront gross resection (IRS group I or II) and the remaining 69\% (159/231) had gross residual disease or biopsy only (Group III) before the initiation of chemotherapy. The 10-year EFS and OS for these patients were similar (Group I, II, III EFS was 79\%, 72\%, 72\%, and OS was $97 \%, 85 \%$ and $92 \%$ respectively, $\mathrm{p}=0.62$ ).

In summary, the evidence would suggest that a conservative approach to the primary surgical intervention in these tumors, when used in conjunction with adequate chemotherapy and possible RT, provides excellent local control and outcomes. This does not imply that upfront resection is unreasonable in very select instances, such as small, localized and well circumscribed tumors that can be grossly excised with minimal compromise to normal local structures. However, the possibility of a complete resection is often difficult to judge so the majority of patients are better served with conservative organ preserving surgical procedures limited to obtaining tissue for diagnosis.

\section{Primary re-excision}

\section{Expert consensus}

Primary re-excision (PRE) in order to achieve complete resection (R0) in patients with macroscopic or microscopic tumor residuals after biopsy or resection, before any adjuvant therapy (IRS Group II or III), is usually not indicated in patients with vaginal RMS.

\section{Evidence}

There is minimal evidence specifically addressing the utility of PRE in this clinical setting. However, the evidence previously discussed regarding primary surgery supports the position that PRE is frequently not 
justified. In the SIOP MMT trial, chemotherapy was commonly used after initial biopsy instead of primary surgery or PRE, and conservative surgery and/or brachytherapy was utilized for patients who failed to achieve CR. This treatment paradigm resulted in excellent outcomes with a 5-year overall survival rate of $91 \%$ and a local failure rate of $18 \%{ }^{4,7}$

\section{Local therapy decisions after induction chemotherapy}

\section{Expert consensus}

Local therapy in its totality is achieved through a combination of chemotherapy, surgical resection and/or radiation (Figure 1). Appropriate chemotherapy can help minimize the need for surgery and radiation, but it does not obviate the need for these local control methods since not all patients will completely respond and others will relapse and thus require increased therapy for salvage.

Fusion-negative, Group I patients should receive appropriate chemotherapy without additional surgery or radiotherapy. Strategies to avoid RT in Group II patients have been investigated both in EpSSG and COG, but the most recent COG protocol ARST0331 was subsequently amended to require 36 Gy RT. Group III patients should undergo induction chemotherapy and then be assessed for response to chemotherapy. COG, EpSSG and CWS protocols mandate tumor re-evaluation after 3 cycles (9 weeks) of chemotherapy. This should include an MRI of the pelvis with contrast media, fat suppression and diffusion weighted imaging as well as cystoscopy, vaginoscopy and bimanual recto-vaginal examination under general anesthesia. Patients with suspected infiltration of the rectum should undergo additional rectoscopy. Patients who have any evidence of disease by either imaging or examination should undergo biopsy.

As mentioned previously, tumors at this site are very sensitive to chemotherapy. However, the need for additional local treatment seems to be significantly impacted by the cumulative dose of alkylating agent in the chemotherapy regimen. When higher cumulative doses of alkylating agents are planned, patients with FOXO1 fusion-negative histology (botryoid, embryonal) with any response after 3 cycles should continue chemotherapy and undergo further reassessment (Figure 2). Local therapy can be potentially omitted if the patient is in complete remission at 6 cycles of chemotherapy. Patients with no response or with residual disease after 6 cycles of induction chemotherapy, or those with unfavorable histology require local therapy with conservative resection and/or radiation. These evaluation points are based on current study group treatment protocols as there are no data supporting the optimal timing for delayed resection. Patients who will receive lower cumulative doses of alkylating agents potentially experience a higher local failure rate and will require local control regardless of response.

In most situations, delayed surgery is limited to biopsy or polypectomy without vaginal wall resection. A biopsy is not recommended in cases of CR during vaginoscopy but is useful in case of suspicious lesions to verify if viable tumor cells are still present. Delayed operations, other than vaginoscopy and biopsy to confirm complete response in patients without radiographic residual disease are rarely indicated. A minority of patients in whom a delayed R0 resection can be achieved may undergo a conservative, but complete tumor resection with organ preservation. For tumors of the upper part of the vagina, partial vaginectomy, partial or total excision of the uterine cervix and trachelectomy (removal of the cervix, surrounding tissue and the upper part of the vagina) are considered organ salvaging procedures. In addition, patients not responding to chemotherapy may require surgical procedures, such as partial or total vaginectomy, for residual tumors. Complete pelvic exenteration is usually not required. If the specimen has tumor free margins, additional RT may be omitted. Care should be taken to avoid injury to the urethra and rectum. Likewise, for patients who fail to achieve CR by the completion of all planned therapy, the role of mass excision is unproven.

Brachytherapy is a preferred form of local treatment. It can be applied as intracavitary and/or interstitial BT. The impact on future fertility should be considered, and a temporary transposition of the ovaries, either laparoscopic or open, may be required for patients undergoing BT if the anticipated ovarian doses exceed tolerance.

Evidence 
Patients with completely resected tumors at diagnosis have a favorable prognosis with chemotherapy alone, i.e. avoiding radiotherapy. In the international pooled analysis, 33 Group I patients were not irradiated and the 10-year EFS was $79 \%$ with an OS of $97 \%{ }^{4}$ The analysis of Group II patients is more difficult as some patients did receive RT and others did not. In general for RMS, the omission of RT for patients with Group II tumors is associated with an increased risk of local failure and lower EFS. ${ }^{10},{ }^{11}$ In the IRS-III trial, Group II patients did receive RT with $41.4 \mathrm{~Gy}$, whereas in COG D9602 $2^{12}$ and COG ART0331 ${ }^{13}$ patients with Group IIA or III vaginal RMS had initial omission of RT if in CR after chemotherapy with or without surgery, but later in the ARST0531 trial, 36 Gy was utilized. In the EpSSG study, patients with unfavourable features (age [?] 10 years and/or tumor size $>5 \mathrm{~cm}$ ) achieving CR and all patients with PR received RT. ${ }^{4}$

Local control of FGU-RMS appears to be additionally influenced by the total cumulative dose of alkylating agents and may impact the amount of local therapy that is required for optimal treatment. Prior to 2004, the COG cumulative cyclophosphamide equivalent doses were higher (24.4-30.8 g/ $\left.\mathrm{m}^{2}\right)$ and RT was infrequently used in Group II and III vaginal RMS tumors. ${ }^{8}$ This resulted in local recurrence rates of $26 \%$ which were higher than local recurrence rates (14\%) for similar tumors at other sites. RT was withheld in accordance with the paradigm utilized in European studies due to the high rate of morbidity associated with pelvic RT in young female patients. After 2004, COG used a lower cumulative cyclophosphamide dose $\left(4.8 \mathrm{~g} / \mathrm{m}^{2}\right)$ in an effort to preserve fertility but there were no changes made in the local control paradigm which still discouraged resection and RT. This lower cyclophosphamide dose treatment regimen had a $43 \%$ local failure rate. However, the use of RT in these patients decreased local failure rates from $53 \%$ to $17 \% .{ }^{14}$ The OS was still excellent for patients with local recurrences since the majority could be salvaged but at the cost of increased chemotherapy, surgical resection and RT. Local recurrences were grossly excised in $73 \%$ of patients. Subsequently, COG ARST1431 is treating patients with Group III FGU-RMS on intermediate risk protocols with a higher cumulative cyclophosphamide dose $\left(8.4 \mathrm{~g} / \mathrm{m}^{2}\right)$ in an attempt to decrease local recurrence as seen on the prior ARST0331. In the large international pooled analysis of FGU-RMS, cooperative group trials that used cumulative cyclophosphamide doses 24.4-30.8 g/ $\mathrm{m}^{2}$ (in COG trials) or cumulative ifosfamide doses of $24-54 \mathrm{~g} / \mathrm{m}^{2}$ (in European trials) were associated with a CR rate of $95 \%$ and local recurrence rate of $24 \%$, with similar rates across cooperative study groups.

All suspicious lesions identified either by imaging or during vaginoscopy require biopsy to confirm resolution of viable tumor. The biopsy is important because an abnormal lesion does not always equate to residual viable tumor. Mature rhabdomyoblasts usually persist following chemotherapy and RT causing an end of therapy mass that can be present on imaging or examination, however, these cells do not predispose to local failure and therefore do not require resection or additional therapy. ${ }^{15}$

There is a role for surgical resection of gross disease provided the tenets of complete gross resection with organ preservation utilizing conservative procedures can be maintained. As mentioned previously in the large multinational study of patients with both vaginal and uterine RMS approximately $31 \%$ of patients were operated on before the initiation of chemotherapy to resect the tumor grossly. ${ }^{4}$ Another $28 \%$ had a surgical resection at some point during initial therapy, for a total of $59 \%$ of patients. Surgery was conservative in $41 \%$ of all patients and radical in $18 \%$. During all primary therapy the frequency of conservative resection (36-53\%) was equally distributed across all multinational study groups and included procedures such as enucleation, partial vaginectomy and partial cervix excision. Fifty-seven percent of patients in the pooled international analysis achieved CR with chemotherapy and biopsy or conservative resection. Radical excision was also equally distributed (8-22\%) among cooperative groups and included procedures such as hysterectomies and vaginectomies. ${ }^{4}$ However, an important caveat is that primary tumor location was the main factor associated with the frequency of radical surgery $(12 / 26$ [46\%] for patients with uterus corpus vs. $25 / 160[16 \%]$, and $6 / 51$ [12\%] for those with vagina and cervix, respectively). Therefore, although much of the resection data presented reflects both vaginal and uterine RMS tumors, it is clear that the need and frequency of radical resections is lower in vaginal tumors compared to uterine tumors that have an inferior EFS and OS. This further supports the good outcomes associated with conservative operative procedures for vaginal RMS tumors. Further supporting this conclusion is a recent review of FGU-RMS using the National Cancer Institute's Surveillance Epidemiology and End Results (SEER) database that determined the OS 
was not improved in patients that had a radical procedure vs a more conservative resection ( $88 \%$ vs $95 \%$ respectively). ${ }^{16}$

\section{Brachytherapy}

Expert consensus

Brachytherapy is a special form of RT with the major advantage of having a small, circumscribed target volume compared to EBRT. Therefore, it affects less healthy tissue leading to reduced functional impairment. Application of BT needs to be adapted to the anatomy of the child. Therefore, individually adapted vaginal molds need to be created since intracavitary BT is the most common modality. Interstitial BT can be used for vulva rhabdomyosarcoma and highly infiltrating tumors may require a combination of interstitial and intracavitary brachytherapy.

When surgeons perform the vaginoscopy after 6 courses of chemotherapy to determine the presence or absence of residual tumor, biopsies or removal of any pedunculated part of the tumor without resecting the vaginal wall can be performed. This can be combined with laparoscopic temporary ovarian transposition and obtaining an impression of the vagina for a mold during the same procedure. The documentation of residual disease should initiate the planning for BT.

Prior to the initiation of BT, treatment planning should be carried out in an interdisciplinary fashion, including radiation oncologists and pediatric surgeons/urologists. Patients can be treated with either lowdose-rate (LDR), pulse-dose-rate (PDR) or high-dose-rate (HDR) BT using iridium-192 ( ${ }^{192} \mathrm{Ir}$ ) or other isotopes. Usually BT is administered as a total dose of 50-60 Gy (LDR and PDR) or 27.5-36 Gy (HDR) over differing timeframes. The residual disease should be considered as clinical target volume for BT but many consider the entire vaginal mucosa at risk for primary vaginal tumors. Definition of the target volume at time of BT is guided by clinical examination under general anesthesia and by any relevant imaging (most frequently MRI). Surgical clips may be placed to help identifying tumors limits on computed tomography. A vaginal impression is useful to guide catheters placement in regard to the target volumes and decrease the irradiated volume.

\section{Evidence}

The development of BT for RMS of the female lower genital tract was first described by Gerbaulet and colleagues and demonstrated preservation of gynecological function. ${ }^{17}, 18$ They found in patients receiving chemotherapy that outcomes with BT was at least as effective as radical surgery (e.g. total vaginectomy; hysterectomy). They applied BT alone or in combination with EBRT. They demonstrated that fertility preservation was feasible with a local control rate of $94 \% .{ }^{17}$ These findings were refined by Martelli et al. demonstrating that patients with complete histologically proven response after chemotherapy did not require further local control, whereas patients with residual disease could be treated by chemotherapy and BT. ${ }^{7,} 18$

Patients can be treated with either low-dose-rate (LDR), pulse-dose-rate (PDR) or high-dose-rate (HDR) BT using iridium-192 $\left({ }^{192} \mathrm{Ir}\right)$ or other isotopes. ${ }^{18,} 19$ PDR BT combines the radiobiological advantages of LDR in terms of organ sparing with the possibility to perform isodoses optimization to improve the therapeutic index. In LDR and PDR BT a total dose of 50-60 Gy (dose rate usually ranging from 0.4 to $0.5 \mathrm{~Gy} / \mathrm{h}$ ) is delivered in 5-6 days and in HDR BT a dose of 27.5-36 Gy is given in 5-12 fractions over 3-10 days.

A major difference between girls treated before and after 1990 was that in the early group the initial tumor extension was included in the BT field, whereas after 1990 only the residual tumor was included in the BT-treated volume. ${ }^{16,18}$ This decrease in the BT-treated volume led to a reduction of acute and long-term sequelae for vaginal or urethral sclerosis or stenosis (from $75 \%$ to $20 \%$ ) while maintaining 5 -year overall survival rate at $91 \%{ }^{16,18}$ Long term sequelae of BT and conservative surgery in the modern era is mainly the risk of vaginal fibrosis and stenosis. Late effects after conservative treatments with BT in early studies were observed in $76 \%$ of the patients and included colorectal, urethral, and ureteral stenosis. ${ }^{20}$ These risks decreased dramatically with advances in treatment planning and the fact that after 1990, only the residual tumor volume was irradiated. ${ }^{21}$ 
There are several techniques described such as development of a vaginal mold from a vaginal impression ${ }^{19}$ or creation of an individual vaginal mold after measurement of the vaginal depth and circumference in the form of an individual hegar dilator with integrated BT tubes. ${ }^{18}$

Rhabdomyosarcoma of the uterus

RMS of the uterus can be found at the cervix uteri or the corpus uteri. The pooled analysis of 237 international patients showed a median age at diagnosis of 2.9 years for uterine primary compared to 13.5 years for cervical primaries. ${ }^{4}$ Additionally, tumors were less frequently located at the cervix $(21 \%)$ or the uterus (11\%) compared to the vagina (68\%). Most of the tumors have FOXO1 fusion negative embryonal histology. ${ }^{4}$ Analysis of the IRS-I to -IV trials revealed a higher incidence for stage IV disease in patients with RMS of the uterus. ${ }^{6}$ Patients with tumors located at the uterus have the worst overall survival in comparison to those located at the cervix or vagina. ${ }^{4}$ Local therapy differs between tumor located at the cervix uteri or the corpus uteri. Therefore, local control is described separately.

Rhabdomyosarcoma of the cervix uteri

\section{Primary surgery and PRE}

Expert consensus

For RMS of the cervix uteri the same principles and expert consensus used for vaginal tumors should be applied. Therefore, patients should undergo cystoscopy, vaginoscopy and bimanual examination under general anesthesia in addition to an MRI scan of the abdomen and pelvis. In cases of suspicious results at the rectum, rectoscopy should be performed. Biopsies should be taken trans-vaginally by polypectomy or incisional / excisional (curettage) biopsy. Partial excision of the cervix for small pedunculated tumors would be considered a conservative resection and should be performed when applicable. Primary radical surgery including hysterectomy or pelvic exenteration are not indicated due to the good response to chemotherapy and improved organ preservation.

Similar to vaginal RMS, PRE should only be undertaken in RMS of the cervix uteri if a complete resection can be achieved in smaller tumors with preservation of organ function.

\section{Evidence}

Before 1972, the treatment of choice was radical surgery followed by chemotherapy and radiotherapy. However, treatment then shifted towards initial biopsy in the IRS-III and -IV trials. ${ }^{22}$ The combination of chemotherapy with delayed conservative surgery with either biopsy or resection but organ preservation resulted in persistent good outcomes and local control. ${ }^{23}$

\section{Local therapy decision}

\section{Expert consensus}

After induction chemotherapy (3 cycles) re-assessment including cystoscopy, vaginoscopy, and bimanual digital rectal examination under general anesthesia is carried out. Additionally, an MRI scan of the abdomen and pelvis is recommended as described for vaginal tumors. Re-assessment is repeated again after 6 cycles of chemotherapy by endoscopy. Follow-up by ultrasound can be carried out in between to assess response.

If there is no visible residual tumor on imaging, endoscopic biopsies of the cervix, with or without hysteroscopy depending on tumor location, should be obtained. This utilization of biopsies even for patients in $\mathrm{CR}$ on imaging is different than the approach for patients with vaginal primaries. If there is no histological evidence of disease, local therapy can be omitted, but patients require a close follow-up (authors opinion: every 3 months for the first 2 years, then every 6 months for the next 3 years). All other cases of uterine RMS require delayed conservative surgical resection and/or RT (with EBRT, proton beam or BT). Residual tumors located at the cervix uteri (especially polypoid lesions) may be treated by partial or total resection of the cervix. Organ preservation is often feasible in combination with BT or EBRT/proton beam therapy. Another surgical option is trachelectomy, in which the cervix and the upper part of the vagina are resected 
en-bloc and an anastomosis between the persisting uterus and vagina is carried out. Trachelectomy can be performed using an open abdominal or laparoscopic approach.

Brachytherapy is carried out as described previously using individual vaginal molds with a brachytherapy tube placed into the cervix uteri. The uterus may not be functional after irradiation, depending on the uterine length and the length of dwell positions within intra-cervical catheter. Ovarian transposition should be offered to patients treated with brachytherapy for cervical disease (see fertility preservation section below).

\section{Evidence}

Direct evidence regarding local control for tumors of the cervix are difficult to find since studies often combine patients with uterine tumors. However, it has been observed that patients with cervical RMS had a trend towards a superior survival rate compared to those with vaginal tumors in the North American SEER database, possibly due to a higher percentage of patients with favorable histology and lower stage tumors. ${ }^{3}$ Residual RMS of the cervix after induction chemotherapy does not seem to require a more radical surgical approach than RMS of the vagina. In the International data analysis only $12 \%$ of patients with tumors at the cervix were treated with radical surgery and this site had a local control rate of $88 \% .{ }^{4}$ Similarily, Martelli et al. demonstrated that the outcome for patients with uterus RMS can be favorable with a high rate of preserved organs. ${ }^{7}$ They demonstrated that 10 of 11 girls with uterine RMS survived and eight of them retained their uterus. Patients with partial resection of the cervix $(n=3)$ or chemotherapy alone $(n=3)$ had little or no local sequelae, but patients undergoing EBRT were found to have long-term functional sequelae. In addition, the local disease management may have had an impact on the outcome since more radical surgical options are available for uterine tumors. ${ }^{3}$ In contrast to the SEER data, Martelli et al. showed that these patients do not do better with more aggressive resections since $65 \%$ of patients achieved complete clinical and radiological remission with chemotherapy alone. ${ }^{7}$ The good outcomes of these children occurred with minimal local control. Nasioudis and colleagues analyzed 70 patients with cervix RMS and also found that there is no survival benefit of extensive surgery since conservative tumor excision can provide adequate local control. ${ }^{16}$

Trachelectomy has been described as an organ-preserving procedure in a small number of children with RMS of the female genital tract. Kayton et al. demonstrated that trachelectomy is technically feasible. ${ }^{24}$ This has also been reported by other groups. ${ }^{15,25} \mathrm{~A}$ major advantage of trachelectomy seems to be the longterm obstectric outcome. Currently, there is no pediatric data on this issue, but trachelectomy is used in adult women with cervical cancer as a fertility preserving procedure for tumors $<2 \mathrm{~cm}$. It has been shown that these fertility preserving procedures resulted in an improved obstetric outcome without compromising oncological safety. The recurrence rate in the literature after this approach for cervical cancer is less than $5 \%$, and $37 \%$ of the women became pregnant with a live birth rate of $67 \%$. Assisted reproductive technologies may also assist these patients that wish to have children. ${ }^{26}$

Complications of RT at this site appear to be more problematic. For children with tumors near the cervix treated with RT, the OS is similar to vaginal RT. However, for children with tumors more near the uterus, $\mathrm{BT}$ of the uterus and cervix with placement of a BT tube into the endometrium can lead to a non-functioning uterus. This complication has also been observed in EBRT of the uterus with more than 20-30 Gy. ${ }^{26}$

Rhabdomyosarcoma of the corpus uteri

\section{Primary surgery and PRE}

\section{Expert consensus}

The same principles should be applied for corpus uteri as proposed for vaginal and cervix uteri RMS. The primary goal is to obtain tissue for diagnosis by appropriate biopsy. Incisional biopsy via laparotomy should be performed in case of tumors extending into the abdominal cavity. An alternative approach is percutaneous sheathed tru-cut biopsy with gelfoam sealing in selected cases. Primary radical surgery including hysterectomy or pelvic exenteration is not indicated. 


\section{Delayed surgery Expert consensus}

Patients in CR after 6 cycles of neoadjuvant chemotherapy do not require secondary tumor resection, but patients with residual tumors after induction chemotherapy located at the uterine corpus should undergo abdominal hysterectomy in order to achieve an R0 resection. Preservation of the distal vagina and the ovaries is usually possible. Oophorectomy is only indicated if there is a gross ovarian involvement by the tumor. Placement of abdominal drains should be avoided. Vaginal hysterectomy is not recommended due to limited intraoperative visualization. RT after hysterectomy is indicated for patients with positive margins after surgery.

\section{Evidence:}

The analysis of the IRS-I to -IV trials showed a trend towards less aggressive surgical approaches from pelvic exenteration in IRS-I towards preservation of a functional genitourinary tract. Therefore, surgical guidelines tended to delay radical surgery in order to avoid it whenever possible. However, in cases in which intensive chemotherapy with or without RT could not eradicate the tumor, radical surgery was used. ${ }^{9}$ In the pooled international analysis definitive local control was achieved in $70 \%$ of uterus RMS, and 7 of 12 patients had hysterectomy as primary surgery. ${ }^{4}$

\section{Local therapy decision}

\section{Expert consensus}

Diagnostic work-up is similar to tumors located at the cervix uteri. An additional MRI of the tumor region is carried out after 6 cycles of chemotherapy.

\section{Management of local relapse}

\section{Expert consensus}

An individual decision must be taken for patients with relapse. Treatment options depend on the previously applied therapy and may consist of radical surgery (hysterectomy, vaginectomy, anterior pelvic exenteration) or radiotherapy (EBRT, proton beam therapy, BT).

\section{Evidence}

In the largest published series, the recurrence rate was $24 \%$ and occurred after a median of 24 months. ${ }^{4}$ Eighty-nine percent of those patients had local relapse and $84 \%$ of relapses had not been previously irradiated. RT appears to confer a protective effect on relapse with only $8 \%$ of relapses occurring after RT, however this impact of RT on EFS did not correlate with OS due to the good response of these tumors to salvage therapy.

\section{Fertility Preservation}

\section{Expert consensus}

A fertility preservation consultation should be initiated prior to therapy. Post-menarchal girls receiving any alkylating agent or pelvic radiation should be offered the option of oocyte harvest if it can be accomplished without inappropriate delay in therapy initiation.

Girls who will receive pelvic radiation may be offered ovarian transposition.

Ovarian tissue cryopreservation (OTC) is an additional, experimental fertility preservation option available to both pre- and post-menarchal girls receiving alkylating agent therapy and/or pelvic radiation. The risk for infertility and premature menopause is proportional to the dose of alkylating agent, as quantified by the cyclophosphamide equivalent dose (CED), but different cooperative study groups and local institutions employ various thresholds for offering this intervention.

Oocyte harvest and cryopreservation in the late teenage years, after therapy completion, is another option.

Evidence 
Girls who undergo therapy for urogenital RMS are at significant risk for future infertility and ovarian insufficiency resulting in premature menopause. Identification of, and subsequent interventions to prevent the complications of premature menopause (osteoporosis, cardiovascular disorders, and impaired psychosocial well-being) are important. ${ }^{27}$

In a large study of survivors of childhood cancer, $38 \%$ of survivors reported having or desiring a pregnancy compared to $62 \%$ of siblings. ${ }^{28}$ Patients and families are interested in being informed about their risk of infertility and discussing fertility preservation options, even when the risk of infertility is relatively modest. ${ }^{29}$ Higher doses of alkylating agents, which form the backbone of chemotherapy regimens for RMS, have been consistently associated with reduced germ cell function and fertility rates. ${ }^{30}$ Furthermore, other components of the multimodal therapy for FGU-RMS can likewise negatively impact future fertility, including pelvic radiation with EBRT or BT, as well as surgical resection of genitourinary organs. In females, a CED of 4-8 $\mathrm{g} / \mathrm{m}^{2}$ was associated with greater than a $2.5 \mathrm{x}$ relative risk of premature menopause, and a CED $>8 \mathrm{~g} / \mathrm{m}^{2}$ with a greater than $4 \mathrm{x}$ relative risk. ${ }^{31}$ However in a long-term study of over 10,000 survivors of childhood cancer, only CED doses in the highest quartile $(>9,639 \mathrm{mg} / \mathrm{m} 2)$ versus no exposure was associated with reduced pregnancy rates. ${ }^{28}$ Therefore, while the doses of alkylating agents given to most children with urogenital RMS impart some risk of future infertility and ovarian insufficiency, further efforts are required to delineate this risk and inform decisions about when to offer fertility preservation procedures.

Oocyte harvest is a non-experimental option available for post-pubertal girls. Random start ovarian stimulation protocols, which no longer require timing with the menstrual cycle, can be performed with minimal delay in treatment initiation. ${ }^{29}$ Laparoscopic OTC is an investigational option for both pre- and post-pubertal girls. Total or partial oophorectomy allows for preservation of ovarian cortical strips for future reimplantation with potential restoration of fertility and hormone function. ${ }^{32}$

While the potential benefit of OTC for girls with RMS of the genitourinary tract is generally driven by the dose of alkylating agent chemotherapy, consideration must also be given to the fertility impact from radiation. The radiation dose to the ovary has a significant effect on future fertility, with one study showing a decrement in the relative chance of pregnancy of 0.18 with doses $>10 \mathrm{~Gy} .{ }^{30}$ The RT doses for RMS, generally ranging from 27-60 Gy regardless of modality, exceed the doses accepted to cause sterilization; 20.3 Gy in infants, 18.4 Gy at age 10 years, and 16.5 Gy at age 20 years. ${ }^{33}$ Depending on their location relative to the tumor and position in the radiation field, the anticipated RT dose to the ovaries should be calculated to assess this risk. Ovarian transposition has an important role for girls receiving pelvic radiation with EBRT or BT. Patients undergoing EBRT require transposition of the ovary to the paracolic gutters with the division of the ligamentous attachments. ${ }^{34}$ In some cases, relocation back to the pelvis after therapy completion is required. Temporary transposition for the 3-10 days of treatment for girls receiving brachytherapy can be achieved without division of the ligamentous attachments. ${ }^{6}$ However, in patients who received pelvic RT for Hodgkin's lymphoma, ovarian transposition did not seem to modify the risk of ovarian insufficiency and further studies are required to assess the long-term benefit of transposition. ${ }^{35}$

\section{Summary of the expert consensus}

The prognosis of RMS arising from the female genital tract is favorable and there is an excellent response to chemotherapy. Initial complete surgical resection is not the goal in RMS of the vagina or uterus. The initial goal of surgery is the establishment of the diagnosis. Vaginal RMS do not require an upfront surgical resection except in very small, localized and well circumscribed tumors that can be completely resected with complete organ preservation. Primary re-excision is usually not indicated in this patient population unless the tumor can be completely resected with complete organ preservation. In tumors that do not completely respond to induction therapy, or who have confirmation of persistent viable tumor identified by screening vaginoscopy with biopsies and are not amenable to surgical resection, local therapy with RT, preferably BT applied as intracavitary and/or interstitial, is helpful. There is a role for surgical resection of gross disease provided the tenets of complete gross resection with organ preservation utilizing conservative procedures can be maintained. 
RMS of the cervix uteri is treated similarly to RMS of the vagina. The diagnosis can be established by transvaginal biopsy or polypectomy. Partial excision of the cervix as primary surgery or PRE is feasible for small pedunculated tumors with preservation of organ function. In patients with evidence of tumor after induction chemotherapy local control is attempted using resection or RT. Brachytherapy is usually carried out with a vaginal mold and an additional brachytherapy tube which is placed into the cervix uteri. Residual tumors at the cervix uteri may be treated by partial or total resection of the cervix. Trachelectomy is an alternative option.

Rhabdomyosarcoma of the corpus uteri is treated following the above-mentioned principles regarding local therapy decision. For patients with persistent tumors after induction chemotherapy, abdominal hysterectomy should be performed.

Fertility preservation should be considered in all patients. The modality depends on the local treatment approach.

\section{REFERENCES}

1. McDowell HP. Update on childhood rhabdomyosarcoma. Arch Dis Child. 2003;88: 354-357.

2. Arndt C, Rodeberg D, Breitfeld PP, Raney RB, Ullrich F, Donaldson S. Does bladder preservation (as a surgical principle) lead to retaining bladder function in bladder/prostate rhabdomyosarcoma? Results from intergroup rhabdomyosarcoma study iv. J Urol. 2004;171: 2396-2403.

3. Kirsch CH, Goodman M, Esiashvili N. Outcome of female pediatric patients diagnosed with genital tract rhabdomyosarcoma based on analysis of cases registered in SEER database between 1973 and 2006. Am J Clin Oncol. 2014;37: 47-50.

4. Minard-Colin V, Walterhouse D, Bisogno G, Martelli H, Anderson J, Rodeberg DA, Ferrari A, Jenney M, Wolden S, De Salvo G, Arndt C, Merks JHM, Gallego S, Schwob D, Haie-Meder C, Bergeron C, Stevens MCG, Oberlin O, Hawkins D; International Society of Pediatric Oncology Sarcoma Committee, the Children's Oncology Group, the Italian Cooperative Soft Tissue Sarcoma Group, and the European pediatric Soft tissue sarcoma Study Group. Localized vaginal/uterine rhabdomyosarcoma-results of a pooled analysis from four international cooperative groups. Pediatr Blood Cancer. 2018;65: e27096.

5. Corpron CA, Andrassy RJ, Hays DM, Raney RB, Wiener ES, Lawrence W, Lobe TE, Maurer HM. Conservative management of uterine pediatric rhabdomyosarcoma: a report from the Intergroup Rhabdomyosarcoma Study III and IV pilot. J Pediatr Surg. 1995;30: 942-944.

6. de Lambert G, Haie-Meder C, Guerin F, Boubnova J, Martelli H. A new surgical approach of temporary ovarian transposition for children undergoing brachytherapy: technical assessment and dose evaluation. J Pediatr Surg. 2014;49: 1177-1180.

7. Martelli H, Oberlin O, Rey A, Godzinski J, Spicer RD, Bouvet N, Haie-Meder C, Terrier-Lacombe MJ, Sanchez de Toledo J, Spooner D, Sommelet D, Flamant F, Stevens MC. Conservative treatment for girls with nonmetastatic rhabdomyosarcoma of the genital tract: A report from the Study Committee of the International Society of Pediatric Oncology. J Clin Oncol. 1999;17: 2117-2122.

8. Walterhouse DO, Meza JL, Breneman JC, Donaldson SS, Hayes-Jordan A, Pappo AS, Arndt C, Raney RB, Meyer WH, Hawkins DS. Local control and outcome in children with localized vaginal rhabdomyosarcoma: a report from the Soft Tissue Sarcoma committee of the Children's Oncology Group. Pediatr Blood Cancer. 2011;57: 76-83.

9. Arndt CA, Donaldson SS, Anderson JR, Andrassy JR, Andrassy RJ, Laurie F, Link MP, Raney RB, Maurer HM, Crist WM. What constitutes optimal therapy for patients with rhabdomyosarcoma of the female genital tract? Cancer. 2001;91: 2454-2468.

10. Million L, Anderson J, Breneman J, Hawkins DS, Laurie F, Michalski J, Rodeberg D, Wharam M, Wolden S, Donaldson SS, Soft Tissue Sarcoma Committee of the Children's Oncology Group. Influence of 
noncompliance with radiation therapy protocol guidelines and operative bed recurrences for children with rhabdomyosarcoma and microscopic residual disease: a report from the Children's Oncology Group. Int J Radiat Oncol Biol Phys. 2011;80(2): 333-338.

11. Schuck A, Mattke AC, Schmidt B, Kunz DS, Harms D, Knietig R, Treuner J, Koscielniak E. Group II rhabdomyosarcoma and rhabdomyosarcomalike tumors: is radiotherapy necessary? J Clin Oncol. 2004;22: 143-149.

12. Raney RB, Walterhouse DO, Meza JL, Andrassy RJ, Breneman JC, Crist WM, Maurer HM, Meyer WH, Parham DM, Anderson JR. Results of the Intergroup Rhabdomyosarcoma Study Group D9602 protocol, using vincristine and dactinomycin with or without cyclophosphamide and radiation therapy, for newly diagnosed patients with low-risk embryonal rhabdomyosarcoma: a report from the Soft Tissue Sarcoma Committee of the Children's Oncology Group. J Clin Oncol. 2011;29: 1312-1318.

13. Walterhouse DO, Pappo AS, Meza JL, Breneman JC, Hayes-Jordan AA, Parham DM, Cripe TP, Anderson JR, Meyer WH, Hawkins DS. Shorter-duration therapy using vincristine, dactinomycin, and lower-dose cyclophosphamide with or without radiotherapy for patients with newly diagnosed low-risk rhabdomyosarcoma: a report from the Soft Tissue Sarcoma Committee of the Children's Oncology Group. J Clin Oncol. 2014;32: 3547-3552.

14. Walterhouse DO, Pappo AS, Meza JL, Breneman JC, Hayes-Jordan AA, Parham DM, Cripe TP, Anderson JR, Meyer HM, Hawkins DS. Reduction of cyclophosphamide dose for patients with subset 2 low-risk rhabdomyosarcoma is associated with an increased risk of recurrence: A report from the Soft Tissue Sarcoma Committee of the Children's Oncology Group. Cancer. 2017;123: 2368-2375.

15. Ortega JA, Rowland J, Monforte H, Malogolowkin M, Triche T. Presence of well-differentiated rhabdomyoblasts at the end of therapy for pelvic rhabdomyosarcoma: implications for the outcome. J Pediatr Hematol Oncol. 2000;22: 106-111.

16. Nasioudis D, Alevizakos M, Chapman-Davis E, Witkin SS, Holcomb K. Rhabdomyosarcoma of the lower female genital tract: an analysis of 144 cases. Arch Gynecol Obstet. 2017;296: 327-334.

17. Flamant F, Gerbaulet A, Nihoul-Fekete C, Valteau-Couanet D, Chassagne D, Lemerle J. Long-term sequelae of conservative treatment by surgery, brachytherapy, and chemotherapy for vulval and vaginal rhabdomyosarcoma in children. J Clin Oncol. 1990;8: 1847-1853.

18. Fuchs J, Paulsen F, Bleif M, Lamprecht U, Weidner N, Zips D, Neunhoeffer F, Seitz G. Conservative surgery with combined high dose rate brachytherapy for patients suffering from genitourinary and perianal rhabdomyosarcoma. Radiother Oncol. 2016;121: 262-267.

19. Gerbaulet A, Haie C, Michel G, Castaigne D, Prade M, Kunkler I, Pomp J, Masselot J, Lhomme C, Chassagne D. Combined radio-surgical treatment in early invasive cervix carcinoma according to prognostic factors. Experience of the Gustave-Roussy Institute. Eur J Gynaecol Oncol. 1992;13: 256-261.

20. Levy A, Martelli H, Fayech C, Minard-Colin V, Dumas I, Gensse MC, Le Deley MC, Oberlin O, HaieMeder C. Late toxicity of brachytherapy after female genital tract tumors treated during childhood: Prospective evaluation with a long-term follow-up. Radiother Oncol. 2015;117: 206-212.

21. Magne N, Oberlin O, Martelli H, Gerbaulet A, Chassagne D, Haie-Meder C. Vulval and vaginal rhabdomyosarcoma in children: update and reappraisal of Institut Gustave Roussy brachytherapy experience. Int J Radiat Oncol Biol Phys. 2008;72: 878-883.

22. Ortega JA. A therapeutic approach to childhood pelvic rhabdomyosarcoma without pelvic exenteration. J Pediatr. 1979;94: 205-209.

23. Rodeberg DA, Stoner JA, Hayes-Jordan A, Kao SC, Wolden SL, Qualman SJ, Meyer WH, Hawkins DS. Prognostic significance of tumor response at the end of therapy in group III rhabdomyosarcoma: a report from the children's oncology group. J Clin Oncol. 2009;27: 3705-3711. 
24. Kayton ML, Wexler LH, Lewin SN, Park KJ, La Quaglia MP, Abu-Rustum NR. Pediatric radical abdominal trachelectomy for anaplastic embryonal rhabdomyosarcoma of the uterine cervix: an alternative to radical hysterectomy. J Pediatr Surg. 2009;44: 862-867.

25. Willows K, Lennox G, Covens A. Fertility-sparing management in cervical cancer: balancing oncologic outcomes with reproductive success. Gynecol Oncol Res Pract. 2016;3: 9.

26. Bouchard-Fortier G, Kim RH, Allen L, Gupta A, May T. Fertility-sparing surgery for the management of young women with embryonal rhabdomyosarcoma of the cervix: A case series. Gynecol Oncol Rep. 2016;18: $4-7$.

27. van Dorp W, Mulder RL, Kremer LC, Hudson MM, van den Heuvel-Eibrink MM, van den Berg MH, Levine JM, van Dulmen-den Broeder E, di Iorgi N, Albanese A, Armenian SH, Bhatia S, Constine LS, Corrias A, Deans R, Dirksen U, Gracia CR, Hjorth L, Kroon L, Lambalk CB, Landier W, Levitt G, Leiper A, Meacham L, Mussa A, Neggers SJ, Oeffinger KC, Revelli A, van Santen HM, Skinner R, Toogood A, Wallace WH, Haupt R. Recommendations for Premature Ovarian Insufficiency Surveillance for Female Survivors of Childhood, Adolescent, and Young Adult Cancer: A Report From the International Late Effects of Childhood Cancer Guideline Harmonization Group in Collaboration With the PanCareSurFup Consortium. J Clin Oncol. 2016;34: 3440-3450.

28. Chow EJ, Stratton KL, Leisenring WM, Oeffinger KC, Sklar CA, Donaldson SS, Ginsberg JP, Kenney LB, Levine JM, Robison LL, Shnorhavorian M, Stovall M, Armstrong GT, Green DM. Pregnancy after chemotherapy in male and female survivors of childhood cancer treated between 1970 and 1999: a report from the Childhood Cancer Survivor Study cohort. Lancet Oncol. 2016;17: 567-576.

29. Oktay K, Harvey BE, Partridge AH, Quinn GP, Reinecke J, Taylor HS, Wallace WH, Wang ET, Loren AW. Fertility Preservation in Patients With Cancer: ASCO Clinical Practice Guideline Update. J Clin Oncol. 2018;36: 1994-2001.

30. Green DM, Kawashima T, Stovall M, Leisenring W, Sklar CA, Mertens AC, Donaldson SS, Byrne J, Robinson LL. Fertility of female survivors of childhood cancer: a report from the childhood cancer survivor study. J Clin Oncol. 2009;27: 2677-2685.

31. Green DM, Nolan VG, Goodman PJ, Whitton JA, Srivastava DK, Leisenring WM, Neglia JP, Sklar CA, Kaste SC, Hudson MM, Diller LR, Stovall M, Donaldson SS, Robison LL. The cyclophosphamide equivalent dose as an approach for quantifying alkylating agent exposure: a report from the Childhood Cancer Survivor Study. Pediatr Blood Cancer. 2014;61: 53-67.

32. Corkum KS, Rhee DS, Wafford QE, Demeestere I, Dasgupta R, Baertschiger R, Malek MM, Aldrink JH, Heaton TE, Weil BR, Madonna MB, Lautz TB. Fertility and hormone preservation and restoration for female children and adolescents receiving gonadotoxic cancer treatments: A systematic review. J Pediatr Surg. 2019; 54(11):2200-09.

33. Metzger ML, Meacham LR, Patterson B, Patterson B, Casillas JS, Constine LS, Hijiya N, Kenney LB, Leonard M, Lockart BA, Likes W, Green DM. Female reproductive health after childhood, adolescent, and young adult cancers: guidelines for the assessment and management of female reproductive complications. J Clin Oncol. 2013;31: 1239-1247.

34. Moawad NS, Santamaria E, Rhoton-Vlasak A, Lightsey JL. Laparoscopic Ovarian Transposition Before Pelvic Cancer Treatment: Ovarian Function and Fertility Preservation. J Minim Invasive Gynecol. 2017;24: 28-35.

35. Fernandez-Pineda I, Davidoff AM, Lu L, Rao BN, Wilson CL, Srivastava DK, Klosky JL, Metzger ML, Krasin MJ, Ness KK, Pui CH, Robison LL, Hudson MM, Sklar CA, Green DM, Chemaitilly W. Impact of ovarian transposition before pelvic irradiation on ovarian function among long-term survivors of childhood Hodgkin lymphoma: A report from the St. Jude Lifetime Cohort Study. Pediatr Blood Cancer. 2018;65(9): e27232. 
Figure 1: Local treatment modalities in RMS of the female GU tract

Figure 2: Treatment algorithm for localized vaginal RMS

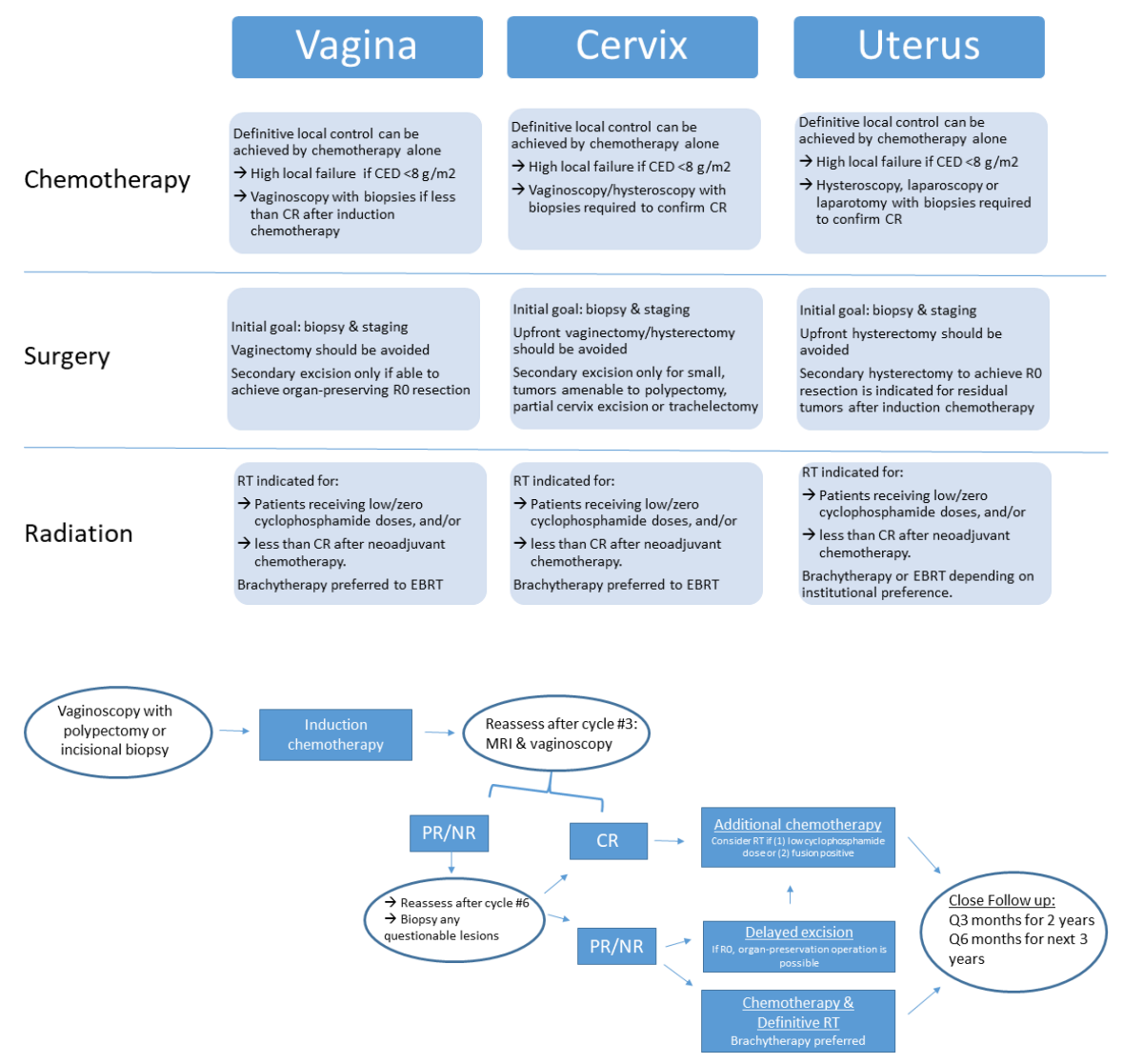

